"(c) 2017 IEEE. Personal use of this material is permitted. Permission from IEEE must be obtained for all other users, including reprinting/ republishing this material for advertising or promotional purposes, creating new collective works for resale or redistribution to servers or lists, or reuse of any copyrighted components of this work in other works. 


\title{
Two-Stage Road Terrain Identification Approach for Land Vehicles Using Feature-Based and Markov Random Field Algorithm
}

\author{
Shifeng Wang ${ }^{1}$, Sarath Kodagoda ${ }^{2}$, Lei Shi ${ }^{2}$, Xiang Dai ${ }^{1}$ \\ ${ }^{1}$ Changchun University of Science and Technology, 7089 Weixing Road, Changchun City, Jilin \\ Province, 130022, China. \\ ${ }^{2}$ University of Technology Sydney, 15 Broadway, New South Wales, 2007, Australia.
}

\begin{abstract}
Road terrain identification is one of the important tasks for driving assistant system or autonomous land vehicle. It plays a key role to improve driving strategy and enhance the fuel efficiency. In this paper, a two-stage approach using multiple sensors is presented. In the first stage, a feature-based identification approach is performed using single-sensor: an accelerometer, a camera, a downward-looking and a forward-looking laser range finders (LRFs), respectively. This produces four classification label sequences. In the second stage, a Majority-Vote is implemented for each label sequences to match them into synchronized road patches. Then a Markov Random Field (MRF) model is designed to generate the final optimized identification results to improve the forward-looking LRF. This approach enables the vehicle to observe the upcoming road terrain before moving onto it by fuses all the classification results using MRF algorithm. The experiments show this approach improved the terrain identification accuracy and robustness significantly for some familiar road terrains.
\end{abstract}

Keywords—road terrain, feature extraction, machine learning, Markov Random Field

\section{Background and Related Work}

In the past decades, driving assistant system and autonomous land vehicle have been developed with achievements worldwide. For the environment perception, knowledge about the road terrain is one of the important information the vehicle should acquire. Different road terrain 
can have a significant impact on vehicle trajectory, velocity and driving acceleration [1]. This paper presents a two-stage method to detect the upcoming road terrain types for a land vehicle.

Numbers of researchers are working on this task using range of sensors and machine learning techniques. An accelerometer was used for a land vehicle [2] while an Inertial Measurement Unit (IMU) was used for a legged robot [3] to extract vibration-based features for the road terrain classifications. Camera was also applied to obtain the colour and textured features for outdoor road terrain identification [4]. The LRF's measurement was also employed with an IMU to improve the classification performance [5]. Although single sensor gives reasonable classification results, multiple sensors were combined to overcome each of the disadvantage under the particular circumstances. Vibration data and image data were used for terrain classification [6]. The images from multiple-view camera and the point cloud from a 3D LRF were incorporated for feature extraction for accuracy improvement of terrain classification in representative environments [7]. In order to estimate the degree of traversability of upcoming terrain, the acceleration features were combined with texture attributes obtained from evaluation of the fractal dimension for function approximation using Gaussian Process regression [8].

Single sensor which could be an accelerometer, camera, and LRF can be used for the terrain classification task. But just as some researchers suggested, each of the sensors had its own shortages and limitations. Hence, multiple-sensor based terrain classification techniques are introduced to overcome the problem. The combinations of accelerometer and camera; the camera and LRF were popularly used. Moreover, most of the researches in the literature were based on small sized robots working at a fixed low speed. The focus of this paper is to devise identification approach for land vehicles operating at a vast range of speeds. It is also intended to

predict the upcoming road terrain types. This needs more specific and intense concentration and further investigations.

\section{Single-Sensor Stage}

Considering that different sensor samples different physical characteristics of the road terrains, accelerometer, camera, and LRF sensors are employed for the terrain classification task, respectively. The experiments using single sensor are then performed and the results are then compared in section 4.3.1. As shown in Figure 1, the experimental platform CAS Research Ute for Intelligence, Safety and Exploration (CRUISE) is a Ford Courier utility vehicle. 


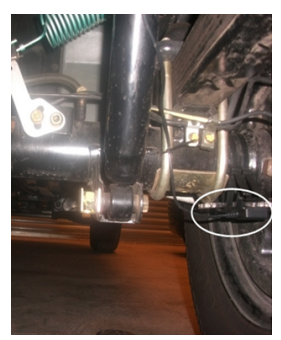

(a) Accelerometer

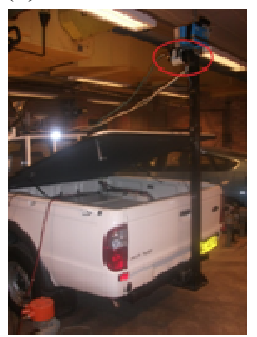

(c) Downward-looking LRF

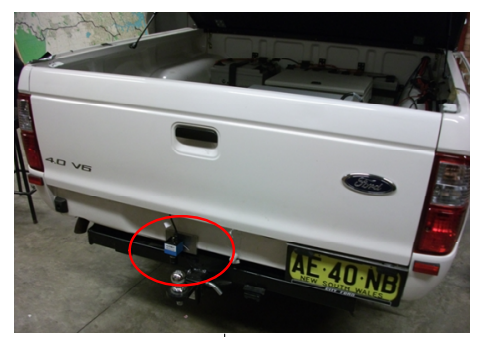

(b) Camera

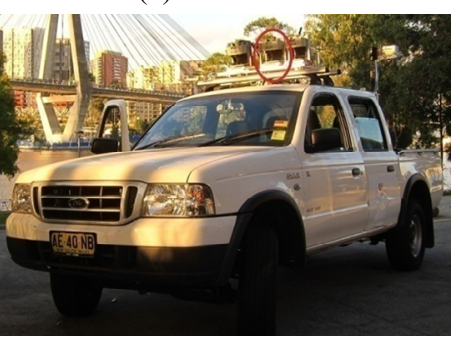

(d) Forward-looking LRF

Figure 1 The mountings of the four sensors

\subsection{Feature Extraction from Acceleration Data}

The accelerometer can be seen in Figure 1 (a) is mounted on the suspension close to the rear-left wheel. The speed data is acquired from a Global Positioning System (GPS) unit. Figure 2 shows the procedure of road profile estimation by using acceleration data, speed data and the quarter vehicle model. According to the previous tests [9], the spatial frequency features are employed rather than other features for classification. The detailed method is described as follows:
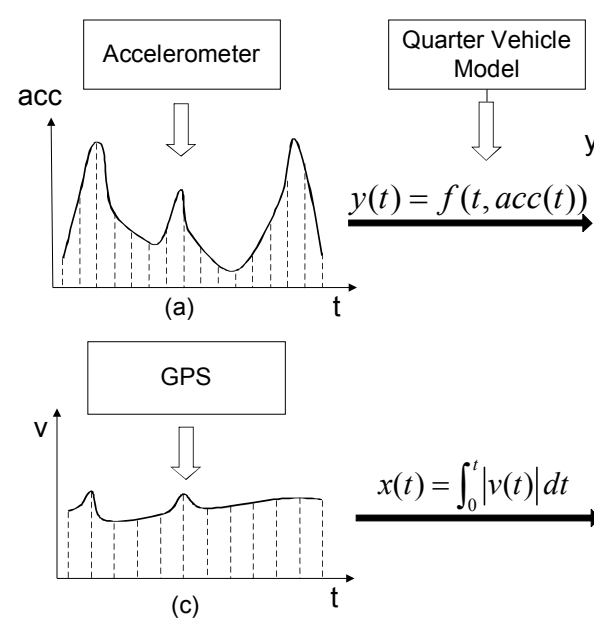
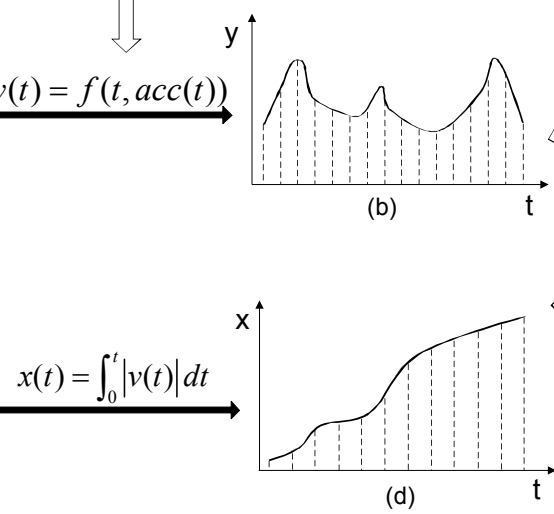
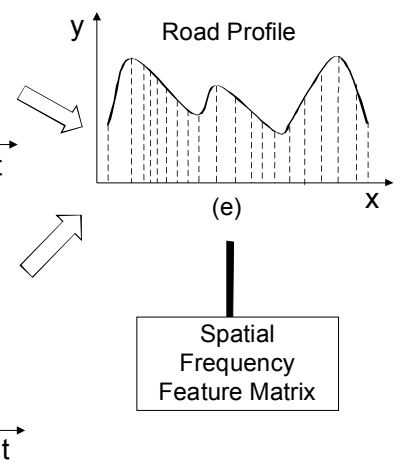

(e)

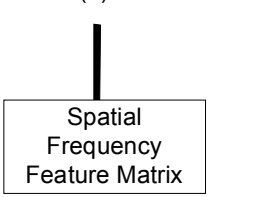

Figure 2 Illustration of spatial frequency feature extraction from road profile 


\subsubsection{From Acceleration (acc-t) to Height (y-t)}

An accelerometer is mounted on the suspension of the vehicle (close proximity to the rear right wheel) to measure the vertical vibration while the vehicle is in motion. Applying the standard quarter car model along with the CRUISE's parameters to the provided time varying raw acceleration data $a c c(t)$ (Figure $2(\mathrm{a})$ ) to calculate out the height of terrain $y(t)$ ( Figure $2(\mathrm{~b})$ ). And this procedure is well described in [9].

\subsubsection{From Speed (v-t) to Displacement $(x-t)$}

As shown in Figure 2 (c), the speed from a GPS unit working at $10 \mathrm{~Hz}$. Low pass filtering is applied to process the un-continuous speed data before for using it. As shown in Figure 2 (d), the vehicle displacement is estimated as:

$$
x(t)=\int_{0}^{t}|v(t)| d t
$$

where $v(t)$ is the measured vehicle speed obtained by the GPS unit. Since the sampling frequency of speed is much lower, a nearest-neighbour interpolation is applied to $x(t)$ data to match the other sensors, including accelerometer, camera, and LRFs.

\subsubsection{Road Profile (y-x)}

It should be noted that the curve $y(t)$ shown in Figure 2 (b) is not the real road profile $y(x)$. Only when the vehicle's speed is constant, the shape of $y(t)$ could be the same with $y(x)$. But the speed of the vehicle keeps changing in most of time. The horizontal distance between every two sampled points is uneven in distance while it is even in time. Therefore, interpolation is employed to $y(x)$ to make the points distributed evenly on a horizontal axis before the FFT implementation.

\subsubsection{Feature Extraction from Road Profile (y-x)}

This road terrain identification system is supposed to report the classification results frequently. The road profile $y$ - $x$ is divided into 4 metres long (length of the vehicle) for each segment. Spatial frequency features from $y$ - $x$ are extracted from the road profile data to form the feature matrix for machine learning. Fast Fourier Transformed, and calculated out the Power Spectral Density (PSD) to form the feature matrix [9]. The parameters of PSD start frequency and end frequency are empirically determined to be 0 to 150 circle/metre with 0.5 circle/metre interval. 


\subsection{Feature Extraction from Image Data}

\subsubsection{Texture of the Terrain Images}

Road terrain images are captured by a camera mounted on the back of CRUISE shooting downward to the road surface, as shown in Figure 1 (c). It is supposed that the texture information on the image is contained in the overall spatial relationship. If the specific angular relationships and distance information between neighbouring pairs of pixels of the image can be described or calculated, then the computed results would present as the texture features. In other words, the formed feature matrix is comprised of spatial dependent grey-level information of nearest neighbours with specified angular intervals. These feature matrices make road terrain type classification possible.

In order to reduce the computing cost, the view field of the camera working at 30 FPS is adjusted to avoid capturing other objects besides the road surface. When CRUISE runs slowly enough, some of the images captured are overlapped to each other. The image process employs the entire image including these overlapped parts. Actually, the terrains would not change frequently if the vehicle moves slowly.

\subsubsection{Feature Extraction}

There are 20 typical texture features are extracted from images to form the feature matrix. These defined features describes the texture of images which are: autocorrelation, contrast, correlation, cluster prominence, cluster shade, dissimilarity, energy, entropy, homogeneity, maximum probability, sum of squares, sum average, sum variance, sum entropy, difference variance, difference entropy, information measures of correlation, inverse difference normalized, and inverse difference moment normalized that can be found in [10].

\subsection{Feature Extraction from Point Cloud Data}

\subsubsection{Geometric Arrangement of the LRF}

Different from the accelerometer which samples and rebuilds the road terrain profile along the vehicle's moving direction, as shown in Figure 3 and Figure 1 (d), a downward-looking LRF can scan road surface vertically to the moving direction. This LRF has a $270^{\circ}$ field of view with $0.5^{\circ}$ angular resolution providing 541 range values per scan, and $50 \mathrm{~Hz}$ sampling rate. While the vehicle is moving forward, it leaves a trace of three dimensional point cloud of the surface. The speed of CRUISE is also obtained from GPS unit. 


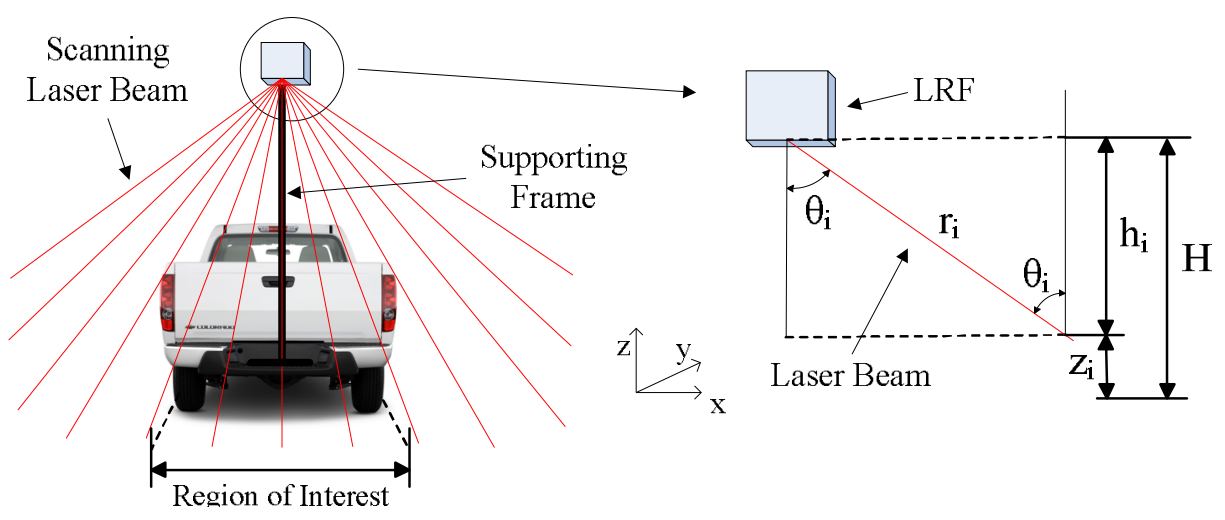

Figure 3 The geometric arrangement of the LRF

\subsubsection{Range Data Processing}

The vertical coordinate, $z_{i}$, of each range measurement seen in Figure 3 can be easily reconstructed by:

$$
z_{i}=H-r_{i} \cos \left(\theta_{i}\right)
$$

where, $r_{i}$ is laser range value, $\theta_{i}$ is included angle between the current laser beam and $z$ axis, and $H$ is the reference height from a relatively flat floor to the height of the LRF. In a similar way, the $\mathrm{x}$-axis coordinate can be calculated as:

$$
x_{i}=r_{i} \sin \left(\theta_{i}\right)
$$

The $270^{\circ}$ scanning field of view contains road surface as well as other nearby objects. Therefore, as illustrated in Figure 3, a $1.3 \mathrm{~m}$ wide region of interest is defined for purposes of this experiment. This brings the distance between two sampling points of a particular scan to approximately two centimetres on a road surface. This LRF is mounted 2.2 meters high above the road surface.

\subsubsection{Feature Extraction}

The estimated 3D surface has more dissimilarity in the resolution along the vehicle moving direction due to the variability of the speed. However, the vehicle speed has minimal effect on the lateral data. Therefore, in this work, only the lateral components are considered. 
The number of scans depends on the vehicle's speed. In general, however, between 35 to 145 scans are captured at speeds of 20 to $80 \mathrm{~km} / \mathrm{h}$ for every 4 metres.

The feature matrix is formed by carrying out the FFT on each scan. The PSD is then calculated in the same way of road profile [11] in section 2.1.4, but the different parameters are set. The start frequency, end frequency and frequency step are decided as 0 to 35 circle/metre with 0.1 circle/metre interval.

\section{Multiple-Sensor Stage Employing MRF Algorithm}

\subsection{Predicting LRF Based Probe}

Obviously, for driver assistant system or autonomous driving, it would be more useful if road terrain type information could be provided before the vehicle moves onto a given road patch. Thus, a forward-looking LRF (LRF1) is now mounted on the roof of the vehicle, as illustrated in Figure 1 (a), to achieve the purpose of predicting the upcoming road terrain in advance.

LRF1 scans the road surface in a two dimensional plane at a $75 \mathrm{~Hz}$ sampling rate. LRF1 has a $100^{\circ}$ field of view with $0.5^{\circ}$ angular resolution providing 201 range values per scan. But only the measurements that laser beam scans on the ground are used in this procedure. The scan data is restricted by the approximate width of the vehicle (Region of Interest in Figure 3).

The only difference between the LRF1 on the roof and the one on the frame looking downward (the one used in the Section 2.3) is that the former one has a scanning tilt angle to the ground while the latter one scans vertically down to the ground. Except a simple calculation to change the range data into the vertical distance via the known tilt angle, the other data processing works are the same as the LRF data processing procedure in Section 2.3. The spatial frequency features is extracted from the pint cloud data to form the feature matrix which is then trained and tested using the Support Vector Machine (SVM) classifier. The classification rate of LRF1, can be seen in Table 1, is much lower than LRF2's. The reason for this is that the tilt angle of LRF1 magnifies the vibration amplitude of LRF1 mounted on the vehicle's roof. This results in too much error of the measurements. Therefore, a Markov Random Field (MRF) algorithm is expected to generate the final optimized results to improve the LRF1.

\subsection{MRF Model}

MRF model is suited to express causal relationships between random variables which stem from the four sensors: a forward-looking LRF (LRF1), an accelerometer (Acc), a camera 
(Cam), and a downward-looking LRF (LRF2). Although each sensor reports a classification result regularly, the reported labels from them are not certain to be the same. That is because the detection capabilities of the sensors are different. Then a simple Majority Vote (MV) [12] algorithm is applied to reduce them into one resulting label for each group. Furthermore, it is expected that the system eventually reports the upcoming road terrain rather than reporting road types after the vehicle has moved on.

\subsection{Nodes in MRF Model}

An undirected graph model is proposed to the observed classification labels from each sensor of the whole system. As shown in Figure 4, the MRF is composed of 5 node types: variables $y, u, w$, and $v$ which denote the classification results from LRF1, Cam, Acc, and LRF2, respectively. The red nodes are unobservable variables $x$ that needs to be 'reset' for the final classification results of LRF1. This reset value is the new value that needs to be assigned using this MRF algorithm. It actually indicates the final terrain types produced by the whole system. Index $i=1, \ldots, \mathrm{n}$ runs over all classification results. Specifically, Index $i$ is the current observed node that needs to be reset while $i-1$ is the former neighbour.

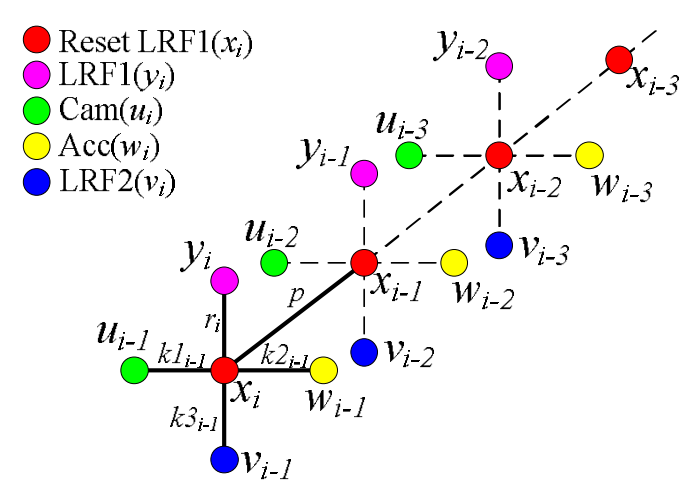

Figure 4 MRF Model for road terrain identification

\subsection{Variable Values of Nodes in MRF Model}

The variable values of the nodes shall be defined for purposes of energy function computation. They should reflect the characteristics of different classes and also have the ability to compute energy function.

The value (binary) of each variable in the MRF algorithm is set as: Asphalt: 1000(B); Concrete: 0100(B); Grass: 0010(B); Gravel: 0001(B). As in many prior tests, the values do not 
work well if they are non-integers, because it is hard to achieve 'equal power' for each class in the energy function. Instinctively, the binary values would lead to an MRF result which is immoderate far from the real value if it is wrong.

\subsection{Clique Potentials in MRF Model}

The mode of the probability distribution defined by the MRF is designed to produce a final road type detection result by fusing the classification results of multiple sensors. The intuition behind the MRF is that the reset label $x_{i}$ is strongly correlated with its corresponding observed label $y_{i}$; three other reported sensor labels; and correlated between its prior reset label $x_{i-1}$. As can be seen from Figure 4, the five clique potentials are defined as:

- $r_{i}$ describes the strength correlation between the reset label $x_{i}$ and its corresponding observed label $y_{i}$;

- $\quad k 1_{i-1}, k 2_{i-1}$, and $k 3_{i-1}$ describe the correlation between $x_{i}$ and $u_{i-1}, x_{i}$ and $w_{i-1}, x_{i}$ and $v_{i-1}$;

- $\quad p$ describes the correlation between $x_{i}$ and $x_{i-1}$.

It should be noted that the clique potentials of $k 1_{i-1}, k 2_{i-1}$, and $k 3_{i-1}$ of the cliques are between $x_{i}$ and $u_{i-1}$, between $x_{i}$ and $w_{i-1}$, and between $x_{i}$ and $v_{i-1}$, but not $u_{i}, w_{i}$, and $v_{i}$. This is because to detect the same patch of road terrain type, Com, Acc and LRF2 act as posterior detectors that the index $i-1$ of theirs are the index $i$ of LRF1 observations. Therefore, to aim at the same patch of the road, the links between $x_{i}$ and $u_{i-1}$, between $x_{i}$ and $w_{i-1}$, and between $x_{i}$ and $v_{i-1}$ are built.

\subsection{Values of Clique Potentials in MRF Model}

The values of the five clique potentials $p, r_{i}, k 1_{i-1}, k 2_{i-1}$, and $k 3_{i-1}$ indicate the correlations between $x_{i}$ that is going to be reset and other labels that help to correct the wrong $x_{i}$ labels. These weighting factors provide the links between central node $x_{i}$ to other nodes. However, those links are not supposed to devote equal weights to the central node. Each link should devote information depending on how 'confident' it is.

The probability of each label resulted from SVM classifier provides the confidence needed. As is known, each classified label produced by the SVM classifier has a probability value. It indicates the confidence in its classification result. Therefore, the classification result probability is utilized as the weighting factor to each clique potential in the MRF energy function. It should be noted that the index of each clique potential needs to be the same as the 
corresponding node's index. Additionally, besides $r_{i}, k 1_{i-1}, k 2_{i-1}$, and $k 3_{i-1}$ which have particular indices to set the values, $p$ that refers to the link between current reset nodes and its former one, is set as constant 1 . The reason is that the current node is strongly relevant to the former one that has already reset by the MRF algorithm.

\subsection{Energy Funtion}

An energy function (potential function) is supposed to be an arbitrary, nonnegative function over a maximal clique. It can be multiplied by any nonnegative functions of subsets of the clique, or equivalently which the corresponding energies can be added in. In this case, this allows setting the particular probability to multiply its corresponding label difference measurement. Specifically, referring to Figure 4, the MRF is defined through the following potentials:

1) Set a function $f(x, y)$ that has output of an arithmetic ' 1 ' or ' 0 ' which depends on the logical operation:

$$
f(x, y)=\left\{\begin{array}{c}
1, x \oplus y=\text { true } \\
0, x \oplus y=\text { false }
\end{array}\right.
$$

2) The clique between the reset classification label (Reset LRF1) and its observed classification label (LRF1):

$$
\Phi=\sum_{i} r_{i} f\left(x_{i}, y_{i}\right)
$$

3) The cliques between the reset classification label (Reset LRF1) and its corresponding other three classification labels (Cam, Acc, LRF2), respectively:

$$
\Lambda=\sum_{i} k 1_{i-1} f\left(x_{i}, u_{i-1}\right)+\sum_{i} k 2_{i-1} f\left(x_{i}, w_{i-1}\right)+\sum_{i} k 3_{i-1} f\left(x_{i}, v_{i-1}\right)
$$

The energy function of the model in this case takes the form:

$$
E(x, y, u, w, v)=\Psi+\Phi+\Lambda
$$

which defines a joint distribution over $x, y, u, w, v$ given by:

$$
p(x, y, u, w, v)=\frac{1}{Z} \exp (-E(x, y, u, w, v))
$$


where $Z$ is a normalization constant (partition function):

$$
Z=\sum_{x, y, u, w, v} \exp (-E(x, y, u, w, v))
$$

\subsection{Optimization}

For the purpose of correctness for the observed classification labels of the LRF1, the reset labels $x$ having a high probability should be found, ideally the maximum probability. This requires the energy function $E(x, y, u, w, v)$ has the lowest value. The iterated conditional modes is used to accomplish this calculation, which is an application of coordinate-wise gradient ascent.

This method first initializes the variables $\left\{x_{i}\right\}$, which are set to $x_{i}=y_{i}$. Then the current observed node $x_{i}$ is selected and the total energy for the four possible states $x_{i}=1000(B)$, $x_{i}=0100(B), x_{i}=0010(B)$, and $x_{i}=0001(B)$ is evaluated, keeping all other node variables fixed. Then $x_{i}$ is set to the state with the lowest energy. This will either leave the probability unchanged, if $x_{i}$ is unchanged, or to lower or increased energy. Because only one variable is changed, this is a computationally cheap local computation. By implementing this MRF algorithm, the classification results of the forward LRF are expected to be improved.

\section{Experiments}

\subsection{Experiment Platform}

The experiment platform CRUISE is equipped with an accelerometer, a camera, two LRFs, a GPS unit, a battery bank, a PC104 computer and a laptop. The two computers communicate with each other via Ethernet. Continuous data can be logged and recorded with timestamp for off-line analysis and processing.

It should be noted that all the feature matrices are normalized to the range of $[0,1]$ before engage them into training/testing stage. Normalization can avoid attributes in greater numeric ranges dominating those in smaller numeric ranges and numerical difficulties during the calculation. 


\subsection{Experiment Implementation}

The experiments were carried out during fine days with average summer temperature and humidity in Great Sydney area, Australia. The CRUISE was driven on four types of road terrains at varying speeds. It includes asphalt terrain at $20-70 \mathrm{~km} / \mathrm{h}$ speed, concrete terrain at 20-40 $\mathrm{km} / \mathrm{h}$, grass terrain at 20-30 km/h, and gravel terrain at speed $20 \mathrm{~km} / \mathrm{h}$, approximately. The data collection also involved the most common road-asphalt terrain including highways, tunnels, and suburb distributor roads. The driving activities also contained many turns, starts, and stops, with no speed restricted.

\subsection{Experiment Results}

Before the terrain two-stage terrain identification experiment, a number of data sets were tested to investigate the optimized classifier and features, and the Principal Component Analysis (PCA) process. According to the prior tests, the SVM classifier was selected for the all training and testing data sets. PCA was tested for finding the best features and contributes to dimensionality reduction. Empirical evaluations lead to the selection of 28 dimensions for acceleration data and 75 dimensions for image data, and no PCA process to point cloud data in many prior tests.

\subsubsection{Feature-Based Classification Experiment}

In the first stage, single-sensor was tested for terrain classification, respectively. A small part of the data sets were used for training and all the others were arranged in collecting sequence.

Table 1 Classification rates of feature-based and MRF-based approaches

\begin{tabular}{|c|c|c|c|c||c|}
\hline & $\begin{array}{c}\text { Asphalt } \\
\text { Terrain }\end{array}$ & $\begin{array}{c}\text { Concrete } \\
\text { Terrain }\end{array}$ & $\begin{array}{c}\text { Grass } \\
\text { Terrain }\end{array}$ & $\begin{array}{c}\text { Gravel } \\
\text { Terrain }\end{array}$ & $\begin{array}{c}\text { Average } \\
\text { Rate }\end{array}$ \\
\hline \hline $\begin{array}{c}\text { Accelero- } \\
\text { meter }\end{array}$ & $17.6 \%$ & $99.6 \%$ & $74.9 \%$ & $85.3 \%$ & $69.4 \%$ \\
\hline Camera & $69.4 \%$ & $99.0 \%$ & $92.5 \%$ & $94.2 \%$ & $88.8 \%$ \\
\hline LRF1 & $44.9 \%$ & $78.2 \%$ & $54.5 \%$ & $94.1 \%$ & $67.9 \%$ \\
\hline LRF2 & $87.9 \%$ & $72.8 \%$ & $83.5 \%$ & $85.1 \%$ & $82.3 \%$ \\
\hline \hline MRF-based & $99.7 \%$ & $99.3 \%$ & $95.5 \%$ & $95.5 \%$ & $97.5 \%$ \\
\hline
\end{tabular}


As shown in Table 1, acceleration-based approach barely worked out on asphalt terrain which is only $17.6 \%$. Actually, asphalt terrain often confused the classifier to be concrete terrain. Expect the asphalt terrain, image-based approach produced very good classification rates which are all higher than $90 \%$. As mentioned in section 3.1, the LRF1 with tilt mounting performance worse than LRF2 with vertical mounting. Because the former one's measurement was ruined by the vehicle's vibration when moving.

\subsubsection{MRF-Based Experiment}

The last row of Table 1 shows the final statistical results of the forward- looking LRF based on multi-sensor fusion. Referring to the row of LRF1, the classification accuracies of all four road types have been increased. The Asphalt road and Concrete road, which are very hard to distinguish, have been classified very well. The performances on Asphalt road and Grass road have been significantly increased by approximately $50 \%$ points. The performance on Gravel road has been slightly increased even though it could be classified well already before MRF fusion was used. The average classification accuracy is consequently increased from $67.9 \%$ to $97.5 \%$. Hence, the forward-looking LRF is now able to accomplish the task of road terrain classification. Its poor prediction accuracy can be significantly improved by employing multi-sensor classification results using the MRF algorithm. Moreover, this classification accuracy $97.5 \%$ (multiple-sensor based using MRF algorithm) is higher than each of $69.4 \%$ (acceleration based), $88.8 \%$ (image based), and $82.3 \%$ (downward LRF based), respectively. This indicates that none of the solo sensor produces better classification accuracy than the multiple-sensor method.

\section{Conclusion}

Due to the shortages and limits of single-based approach, a multiple-sensor fusion approach using MRF algorithm was presented to improve the terrain classification accuracy for land vehicles. The MRF algorithm was employed to fuse all the sensors' classification results. An MRF model that contained five types of nodes and five cliques which described the relationships between the classification results of the accelerometer, the camera, and the two LRFs was designed. By defining the variable values of nodes and the values of clique potentials, the energy function in this case was then created. The optimization computing method was introduced to solve the energy function. The comparison results between the predicting LRF and its corresponding MRF's showed that the MRF-based multiple-sensor fusion approach was extremely effective and robust to accomplish the task of the road terrain classification. However, there could be some other sensors involved or replacements. For example, a camera producing 
greater image quality mounted on the roof of the vehicle could be the second sensor that predicts the upcoming road terrain besides the forward LRF. The future work is also suggested to compare other classification result fusion method with this MRF based one.

\section{Acknowledgements}

This work is funded by National Natural Science Foundation of Jilin Province (20150101047JC), Changchun University of Science and Technology for Young Scholar (XQNJJ-2014-05), Funding of Science and Technology Commission of Shanghai Municipality (13dz2260100), National Key Basic Research Program of China (2014CB744200), and Open Projects of Shanghai Key Laboratory of Deep Space Exploration Technology (DS201509-002, DS201507-001). This work is also supported by the Centre for Autonomous Systems of the University of Technology Sydney.

\section{References}

[1] R. Ghandour, A. Victorino, A. Charara, D. Lechner, "Risk Indicators Evaluation Based on Anticipated Vehicle Dynamics Parameters", IEEE Intelligent Systems, Vol. 27, No 2, March-April 2012, pp. 68-73.

[2] C. C. Ward, K. Iagnemma, "Speed-independent vibration-based terrain classification for passenger vehicles”, Vehicle System Dynamics, Vol. 47, No 9, September 2009, pp. 1095 1113.

[3] S. Manjanna, G. Dudek, P. Giguere, "Using Gait Change for Terrain Sensing by Robots", International Conference on Computer and Robot Vision (CRV), Regian, Canada, $28^{\text {th }}-$ $31^{\text {st }}$, May 2013. pp. 16-22.

[4] S. M. Abbas, A. Muhammad, S. A. Mehdi, K. Berns, "Improvements in Accuracy of single Camera Terrain Classification”, International Conference on Advanced Robotics (ICAR), Montevideo, Uruguay, $25^{\text {th }}-29^{\text {th }}$, November 2013, pp. 1-6.

[5] D. Wang, L. Du, H. He, "Terrain Recognition Improves the Performance of NeuralMachine Interface for Locomotion Mode Recognition", International Conference on Computing, Networking and Communications (ICNC)), San Diego, US, $28^{\text {th }}-31^{\text {st }}$, January 2013, pp. 97-91. 
[6] K. Otsu, M. Ono, T. Fuchs, I. Baldwin, T. Kubota, "Autonomous Terrain Classification with Co- and Self-Training Approach", IEEE Robotics and Automation Letters, No 9, February 2016, pp. 1.

[7] S. T. Namin, M. Najafi, L. Petersson, "Multi-View Terrain Classification Using Panoramic Imaery and LIDAR", IEEE/RSJ International Conference on Intelligent Robots and Systems (IROS 2014), Chicago, US, $14^{\text {th }}-18^{\text {th }}$, September 2014, pp. 14-18.

[8] Mohammed Abdessamad Bekhti, Yuichi Kobayashi, "Prediction of Vibrations as a Measure of Terrain Traversability in Outdoor Structured and Natural Environments", Image and Video Technology, Vol. 9431, February 2016, pp. 282-294.

[9] S. Wang, S. Kodagoda, R. Khushaba, "Towards Speed-Independent Road-Type Classification" International Conference on Control Automation Robotics \& Vision (ICARCV 2012), Guangzhou, China, $5^{\text {th }}-7^{\text {th }}$, December 2012, pp. 614-619.

[10] D. Clausi, "An Analysis of Co-Occurrence Texture Statistics as a Function of Grey Level Uantization”, Remote Sensing, Vol. 28, No 1, 2002, pp. 45-62.

[11] S.Wang, Sarath Kodagoda, Ravindra Ranasinghe, "Road Terrain Type Classification based on Laser Measurement System Data", Australasian Conference on Robotics and Automation (ACRA), Wellington, New Zealand, 3rd-5th, December 2012.

[12] E. Bauer, R. Kohavi, "An Empirical Comparison of Voting Classification Algorithms: Bagging, Boosting, and Variants", Machine Learning, Vol. 36, No 1/2, July/August, 1999, pp. 105-139.

\section{Biographies}

Shifeng Wang, an Associate Professor of Changchun University of Science and Technology, 130022, China. His research interests are Environmental Perception, Opto-Electronics Detection, and Machine Learning. He received his PhD degree at University of Technology Sydney, Australia in 2013. His email: SF.Wang@cust.edu.cn.

Sarath Kodagoda, an Associate Professor of University of Technology Sydney, 2007, Australia. He received his $\mathrm{PhD}$ degree at Nanyang Technological University, Singapore in 2004. His 
research interests are Robotics, Human Robot Interaction, Driver Assistance Systems. He is member of IEEE Robotics and Automation Society and IEEE Communications Society. His email: Sarath.Kodagoda@uts.edu.au.

Lei Shi, a Research Associate of University of Technology Sydney, 2007, Australia. He received his PhD degree at University of Technology Sydney, Australia in 2013. His research interests are Machine Learning, Spatial Statistics, Sensor and Signal Processing. His email: Lei.Shi$1 @$ uts.edu.au.

Xiang Dai, an undergraduate student of Changchun University of Science and Technology, 130022, China. His research interest is Opto-Electronics Detection and Cloud Point data Process. His email: DinoDai68@yahoo.com. 